\title{
Deux disques de musique peul
}

\section{François Borel}

\section{(2) OpenEdition}

\section{Journals}

Édition électronique

URL : http://journals.openedition.org/ethnomusicologie/2367

ISSN : 2235-7688

Éditeur

ADEM - Ateliers d'ethnomusicologie

Édition imprimée

Date de publication : 1 janvier 1989

Pagination : 304-307

ISBN : 2-8257-0178-5

ISSN : $1662-372 X$

Référence électronique

François Borel, « Deux disques de musique peul », Cahiers d'ethnomusicologie [En ligne], 2 | 1989, mis en ligne le 15 septembre 2011, consulté le 19 avril 2019. URL : http://journals.openedition.org/ ethnomusicologie/2367

Ce document a été généré automatiquement le 19 avril 2019

Article L.111-1 du Code de la propriété intellectuelle. 


\title{
Deux disques de musique peul
}

\author{
François Borel
}

\section{RÉFÉRENCE}

Guinée : les Peuls du Wassolon - la danse des chasseurs. Enregistrements : Patrick Larue ; textes, traduction et commentaires : Patricia Pailleaud, Daniela Langer et Abdoulaye Diarra. 1 CD (DDD) OCORA C 558679, 1987. En coédition avec Les Films du Village. Nomades du désert : les Peulhs du Niger. Enregistrements, textes et production : Roselyne François et Manuel Gomes. 1 CD Playa Sound PS 65009, [1987].

Guinée : les Peuls du Wassolon - la danse des chasseurs. Enregistrements : Patrick Larue ; textes, traduction et commentaires : Patricia Pailleaud, Daniela Langer et Abdoulaye Diarra. 1 CD (DDD) OCORA C 558679, 1987. En coédition avec Les Films du Village.

Nomades du désert : les Peulhs du Niger. Enregistrements, textes et production : Roselyne François et Manuel Gomes. 1 CD Playa Sound PS 65009, [1987].

1 Ces deux disques, publiés en 1987, ont un seul point commun, qui justifie d'ailleurs ce compte rendu : ils illustrent, chacun à sa manière, la diversité de l'univers musical d'une ethnie dispersée du Sénégal au Lac Tchad, en majorité dans la frange sahélienne de l'Afrique de l'ouest. Parmi les groupes peuls, seuls quelques-uns sont restés nomades. C'est le cas des Wodaabe du Niger. Les autres se sont petit à petit assimilés à des degrés divers aux populations sédentaires dont ils ont adopté certains traits culturels, notamment la musique.

2 La musique des chasseurs-griots peuls du Wassolon (nord-est de la Guinée) ne semble pas se distinguer de celle des Malinké dont ils ont adopté la langue. Comme le précise le texte accompagnant le disque, et même si l'usage demeure de les appeler «Peuls du Wassolon ", leurs origines restent obscures et, dans cet état d'incertitude, on peut se 
demander s'il n'aurait pas été plus judicieux d'attribuer le nom de Malinké à cette population, comme l'ont fait Gilbert Rouget pour ses enregistrements de $1952^{1}$ et Michel de Lannoy dans son disque consacré aux Sénoufo ${ }^{2}$. Il est probable qu'actuellement, le qualificatif «Peul » constitue un meilleur argument de vente que celui de « Malinké ».

3 Les dix plages du disque sont entièrement consacrées à des chants de griots qui s'accompagnent sur de grandes harpe-luths (de type kord) à six cordes réparties sur deux plans parallèles $(3+3)$. Cet instrument, appelé bourounouba, joue un ostinato mélodicorythmique en compagnie d'instruments idiophones tels que le racleur métallique karanyan, des hochets de nature indéterminée et du bruissement métallique permanent entretenu par le vibrateur fiché dans le manche de l'instrument et que les auteurs appellent « un vacarme délicatement maitrisé » (p. 4). Parfois, cette combinaison résulte en un subtil mélange de rythmes binaires et ternaires. Le chanteur soliste alterne voix de tête et récitatifs lorsqu'il énumère, sur un débit extrêmement rapide, les noms de chasseurs ou de forgerons célèbres. Il est parfois remplacé par le son fluet d'un sifflet de chasse simbon qui intervient hors de toute contrainte rythmique. Le chœur, apparemment constitué de trois autres chanteurs-instrumentistes, lui répond à l'unisson et souligne certaines de ses phrases par des interpellations exaltées. S'y adjoignent à l'occasion les voix de jeunes filles, notamment durant les séquences de danse.

4 La traduction des chants figure en partie sur la brochure du disque avec quelques explications : il s'agit de louanges et de récits sur le monde mythique des animaux et des chasseurs. A part l'illustration de couverture, qui présente deux griots anonymes et leur instrument, la brochure contient encore quelques photos, non légendées et sans rapport avec le contenu du disque, notamment de tambourinaires et de danses de jeunes filles, ce qui laisse supposer que les tambours font aussi partie de cette musique de chasseurs,

5 La qualité des enregistrements, effectués à l'aide de matériel numérique, est irréprochable (DDD). Toutefois, le souci de ne pas séparer les plages par des silences a peut-être conduit les auteurs à une certaine artificialité que trahit parfois un montage approximatif. C'est le cas entre les plages 1 et 2,4 et 5, 9 et 10 . Par ailleurs, la plage 10 présente un défaut de montage à $0^{\prime} 25^{\prime \prime}$.

6 Malgré le «coup de foudre» (p. 3) des auteurs du disque et leur louable intention de respecter l'unité de la musique des chasseurs wassolonké qui «s'impose grâce à l'exquise répétitivité de leurs chants » (id.), on peut regretter qu'ils n'aient pas réservé une place à une autre forme musicale de ce groupe. Il est, certes, tout aussi louable de leur part d'avoir délibérément renoncé à présenter une mosaïque d'exemples musicaux, toujours frustrants par leur brièveté. D'un autre côté, il est évident que jamais un disque, si long soit-il, ne parviendra à restituer l'intégralité d'une forme musicale. Si les cinq premières plages de ce compact sont suffisamment riches en variations de formules mélodicorythmiques pour captiver le profane, la seule lecture des traductions ne saurait suffire à tenir en haleine l'auditeur pendant les 68 minutes que durent les dix chants dont la tonalité ne varie pratiquement pas. Ou alors, il suffit tout simplement de se laisser bercer par cette répétitivité et cette continuité, bref, davantage " entendre » qu'« écouter ».

7 Contrairement aux Peuls du Wassolon, les Wodaabe du Niger (appelés abusivement bororos) sont des nomades qui se déplacent par petits groupes tout au long de l'année avec leurs troupeaux de zébus à longues cornes bororodji, à la recherche de pâturages favorables. On les rencontre fréquemment aux marchés de brousse où ils viennent troquer leurs produits laitiers contre des céréales, ou tout simplement se retrouver de manière informelle entre classes d'âge pour échanger les nouvelles, danser et chanter. A 
chaque hivernage (saison des pluies : juillet-août), les différents groupes se réunissent et organisent de grandes fêtes de retrouvailles au cours desquelles ils réaffirment par la danse leur identité communautaire. C'est aussi à cette occasion que se pratiquent les échanges matrimoniaux entre lignages.

Le disque dont il est question ici nous offre un échantillon des types de chants d'hommes et de femmes exécutés au cours de ces danses de séduction, tout en nous donnant un aperçu de la vie rurale et de la modeste musique instrumentale de ces pasteurs nomades.

L'intérêt des chants wodaabe réside dans leur polyphonie, qui les distingue de tous les autres chants collectifs sahéliens. Comme l'a décrit Zygmunt Estreicher ${ }^{3}$ (auquel les auteurs ont vraisemblablement emprunté quelques lignes), le mécanisme de cette polyphonie « résulte du concours de trois procédés : le chant simultané de deux variantes d'une même mélodie [...], l'empiètement de deux phrases consécutives de la mélodie l'une sur l'autre (tuilage) [...]; enfin la répartition des degrés consécutifs d'une mélodie entre plusieurs voix qui, au lieu de se relayer simplement, s'attardent chacune sur son degré " (p. 74). Cette technique particulièrement complexe, qui laisse croire à la présence d'un bourdon, est illustrée sur cinq des quinze plages du disque $(2,6,7,9,15)$, alors que trois autres documents $(1,4,13)$ nous livrent des versions solistes de ces mêmes chants, isolant ainsi la partie du "coryphée " dont le choeur reprend les derniers mots de chaque phrase. Trois exemples de choeurs de jeunes filles $(5,8,10)$, à caractère responsorial plus conventionnel, complètent cet échantillonnage de musique vocale.

Les rares instruments de musique caractéristiques de la vie nomade pastorale sont représentés par la flûte (11), appelée cecerou (terme probablement apparenté à la tasinsikh des bergers touaregs ou à la sarewa des Haoussa), qui est un tuyau à quatre trous (et non cinq ou six), à embouchure simple, tenu obliquement (et non droit), dont la sonorité et le jeu ne la différencie pas de celle des Touaregs ; par le chalumeau ou la clarinette (et non hautbois) odiliarou (3), fait d'une tige de mil et dont l'embouchure est constituée d'une anche partiellement découpée dans l'écorce même de la tige. A noter que, chez les éleveurs nomades, la flûte est en général jouée par les gardiens de vaches, alors que le chalumeau est plutôt un instrument d'enfant également consacré au gardiennage des moutons. Le troisième instrument est un arc musical à calebasse kitimggama dont l'extrait qui nous est offert (14) n'est malheureusement pas très convaincant. Il est vraisemblable que cet instrument a été emprunté aux populations sédentaires, à l'instar du fadengama des Touaregs. La vie des bergers est encore illustrée par un exemple d'appels au bétail d'un jeune berger (12).

11 Comme ils le précisent dans une autre publication ${ }^{4}$, les auteurs sont journalistes, reporters-photographes et auteurs-conférenciers. En outre, ils ont longuement partagé la vie des Wodaabe et appris leur langue. Malgré ces atouts, il faut malheureusement déplorer le manque d'informations sur les enregistrements, ainsi que plusieurs lacunes ou erreurs. Tout d'abord, précisons que ces Wodaabe ne vivent pas dans le désert, comme le laisse supposer le titre, mais dans une zone aride appelée "steppe arbustive clairsemée » par les géographes; ensuite, que la graphie peulh (avec un «h») a été abandonnée depuis longtemps ; de plus, que tous les Peuls du Niger ne sont pas nomades; et enfin qu'en anglais, «Peuls » se traduit par « Fulani». On aurait souhaité davantage de précisions sur les danses comme le «ruumi», le «yake» et le "gerewol»: leur déroulement et leur hiérarchie, ainsi qu'une traduction, au moins partielle, des textes chantés; on manque aussi d'informations sur l'identité des lignages et des clans rencontrés et enregistrés. Par ailleurs, il semble qu'il y ait quelques confusions dans 
l'intitulé des chants : le chant 6 « Lelore » n'est-il pas «Barka morada » (7), qui lui-même est peut-être un « ummalee » (2) ? Et le «Chant de Djaoma », n'est-il pas aussi un chant de « ruumi » ou un «Lelore »? Même si les publications n'abondent pas sur la musique des Wodaabe, on aurait pu s'attendre à plus de rigueur de la part de journalistes qui ont partagé la vie de ce groupe.

La qualité des enregistrements (analogiques ou numériques?) est satisfaisante, à part l'effet de "compression» audible lors des battements de mains dans la plage 2 et provoqué sans doute par un enregistrement sur « automatique ».

13 Mais c'est surtout le montage qui a été négligé : un bon tiers des plages $(1,5,6,7,9,15)$ débutent ou finissent brutalement, au beau milieu de l'enregistrement. C'est aussi le cas pour les chants de jeunes filles, qui se succèdent abruptement sur la plage 10, entrecoupés de dialogues.

Bref, ces magnifiques chants wodaabe méritent mieux que la désinvolture avec laquelle ce CD a été publié.

\section{NOTES}

1. Musique d'Afrique occidentale, Vogue LDM 30116, A6 : «Chant pour la danse des chasseurs » et Musique malinké - Guinée, Vogue LDM 20113, B6 : «Chant pour la danse des chasseurs ».

2. Côte d'Ivoire, Sénoufo: musique des funérailles fodonon. 1 disque $33 \mathrm{t} .30 \mathrm{~cm}$, Le Chant du Monde LDX 74838, Coll. du Musée de l'Homme/CNRS, 1984, B4 : « Musique de chasseurs malinké ».

3. Zygmunt Estreicher : "Chants et rythmes de la danse d'hommes bororo ", Bulletin de la Société neuchâteloise de géographie 51(5), 1954-55 : 57-93.

4. MALIKI Angelo, FRANÇOIS Roselyne et GOMES Manuel: Nomades peuls. Paris : L'Harmattan, 1988. $72 \mathrm{p}$. 